Article

\title{
An Optimal Decision Rule for a Multiple Selling Problem with a Variable Rate of Offers
}

\author{
Georgy Sofronovid \\ Department of Mathematics and Statistics, Macquarie University, Sydney, NSW 2109, Australia; \\ georgy.sofronov@mq.edu.au
}

Received: 10 March 2020; Accepted: 21 April 2020; Published: 2 May 2020

\begin{abstract}
An asset selling problem is one of well-known problems in the decision making literature. The problem assumes a stream of bidders who would like to buy one or several identical objects (assets). Offers placed by the bidders once rejected cannot be recalled. The seller is interested in an optimal selling strategy that maximizes the total expected revenue. In this paper, we consider a multi-asset selling problem when the seller wants to sell several identical assets over a finite time horizon with a variable number of offers per time period and no recall of past offers. We consider the problem within the framework of the optimal stopping theory. Using the method of backward induction, we find an optimal sequential procedure which maximizes the total expected revenue in the selling problem with independent observations.
\end{abstract}

Keywords: dynamic programming; sequential decision analysis; optimal stopping; multiple stopping rules; asset selling

MSC: 60G40; 62L15; 62P20; 91B26

\section{Introduction}

Assume we sequentially observe independent identically distributed random variables $y_{1}, y_{2}, \ldots, y_{N}$, where the value $y_{n}$ can be interpreted as the value of an asset (for example, a house) at time $n$. We have $K$ identical objects, which we want to sell within the finite time horizon $N$, and at each time $n$ we may receive no more than $C_{n}$ offers, and we are not allowed to recall past offers. Our decision to sell $c_{n}, 0 \leq c_{n} \leq C_{n}$, objects at time $n$ solely depends on the information we know already and it does not depend on the observations we have not seen yet. If we decide to sell at times $m_{1}, m_{2}, \ldots, m_{k}, 1 \leq m_{1}<m_{2}<\cdots<m_{k} \leq N, 1 \leq k \leq K$, we get a gain

$$
Z_{m_{1}, m_{2}, \ldots, m_{k}}=c_{m_{1}} y_{m_{1}}+c_{m_{2}} y_{m_{2}}+\cdots+c_{m_{k}} y_{m_{k}}
$$

where $c_{m_{1}}+c_{m_{2}}+\cdots+c_{m_{k}}=K, 0 \leq c_{n} \leq C_{n}, C_{n}$ are known positive constants, $n=1,2, \ldots, N$. In the other words, $C_{n}$ is the demand and $y_{n}$ is the price of the asset at time $n$. The problem consists of finding a sequential procedure for maximising the expected gain. Since $c_{n}$ are not fixed, the problem we consider in this paper becomes much more complicated compare to the problem with a fixed rate of offers $C_{n}=C, n=1,2, \ldots, N$, studied in [1].

The multiple selling problem described above, which belongs to a class of sequential decision problems, can be considered within the framework of the optimal stopping theory [2] and its extension, the theory of optimal multiple stopping rules [3-5]. Various applications of optimal stopping problems can be found in many fiellds including job search [6,7], engineering [8,9], games [10], resource allocation [11], and insurance [12,13]. Different multiple selling problems have been considered in the literature. Multiple-stopping problems with random horizon are considered in [14]. 
In [15], the author studies a multi-person stopping game with players' priorities. In [16], the author considers a problem of selling several identical commodities in a finite time interval with potential customers arrive according to a Poisson process. In [13], the authors apply optimal multiple stopping rules to analyse a class of insurance products where the policy holder has the right to insure several of its annual operational losses in a finite time horizon. The general formulation of a discrete time optimal stopping problem under the criterion of a fractional reward is given in [17]. A problem of selling several identical objects with a finite horizon and with one offer per time period, $C_{n}=1$, and no recall of past offers is discussed in [18]. This problem is generalised in [1], where we consider a model with a fixed number of offers per time period, $C_{n}=C$. The methodology of optimal multiple stopping rules also allows us to solve problems with different types of actions, for example, buying and selling. In [19], we propose a buying-selling problem with independent observations, where we need to make two optimal stops. This problem is generalised in [20] to a case when we can make more than two stops. In [21], we generalise the model considered in [19] to a case of dependent random variables.

Many authors have considered different asset-selling problems, which are sometimes called house-selling problems (for example, see [22]). In [23], the authors consider the problem of selling more than one object with single offer per day and recall of past offers is allowed. The probelm of selling non-identical objects with one offer per time period is studied in [24]. In [25], the authors generalise the house-selling problem to the problem with vector offers. Two different versions of the selling problem under both finite and infinite horizons are proposed in [26]. In the first version, only the most recently obtained offer may be accepted with no recal allowed, while in the second version, any previously received offer may be accepted with recall allowed. In [27], the authors study asset-selling problems with holding costs. In [28], the authors propose a non-parametric approach to a buying-selling problem with the finite time horizon.

The paper is structured as follows. Section 2 includes some results of the theory of optimal multiple stopping rules. In Section 3, we find an optimal multiple stopping rule for the selling problem. The proof of the result is based on backward induction. Section 4 presents two examples illustrating how the optimal procedure works. Section 5 concludes the paper with a short discussion.

\section{Optimal Multiple Stopping Rules}

\subsection{Definitions}

Let $y_{1}, y_{2}, \ldots$ be a sequence of random variables, which we observe one by one. If we stop at time $m_{i}$, after having observed $y_{1}, \ldots, y_{m_{i}}, i=1, \ldots, k$, we select the corresponding values $y_{m_{1}}, \ldots, y_{m_{k}}$.allowed After stops at times $m_{1}, \ldots, m_{k}$, we receive a gain $Z_{m_{1}, \ldots, m_{k}}$, which is a function of the selected observations $y_{1}, \ldots, y_{m_{k}}$. For example, $Z_{m_{1}, \ldots, m_{k}}$ may be the sum of the selected values, that is, $Z_{m_{1}, \ldots, m_{k}}=y_{m_{1}}+\cdots+y_{m_{k}}$ (e.g., see $\left.[1,18]\right)$. Another example is a buying-selling problem with two stops allowed (see $[19,21]$ ), when $Z_{m_{1}, m_{2}}=y_{m_{2}}-y_{m_{1}}$. For other examples of the gain function, see $[13,20]$. In this paper, the gain function takes the Form (1).

We assume that the mathematical framework consists of

1. a probability space $(\Omega, \mathcal{F}, \mathbf{P})$;

2. a non-decreasing sequence $\left\{\mathcal{F}_{n}, n \geq 1\right\}$ of the $\sigma$-algebras such that $\mathcal{F}_{m_{1}} \subseteq \cdots \subseteq \mathcal{F}_{m_{k}} \subseteq \mathcal{F}$ for all $1 \leq m_{1}<\cdots<m_{k}$

3. a random process $\left\{Z_{m_{1}, \ldots, m_{k}}, \mathcal{F}_{m_{k}}, m_{k}>m_{k-1}\right\}, Z_{m_{1}, \ldots, m_{k}}$ is $\mathcal{F}_{m_{k}}$-measurable, for each fixed integer $m_{1}, \ldots, m_{k-1}, 1 \leq m_{1}<m_{2}<\cdots<m_{k-1}$.

It is clear that within this framework our decision at time $n$ depends only on the observed values $y_{1}, \ldots, y_{n}$ and we are not allowed to stop twice at the same time, $1 \leq m_{1}<\cdots<m_{k}$. These assumptions are stated in the definition of a multiple stopping rule below.

Definition 1. A collection of integer-valued random variables $\tau=\left(\tau_{1}, \ldots, \tau_{k}\right)$ is called a multiple stopping rule if the following conditions hold: 
1. $\left\{\omega \in \Omega: \tau_{1}(\omega)=m_{1}\right\} \in \mathcal{F}_{m_{1}}, \ldots,\left\{\omega \in \Omega: \tau_{1}(\omega)=m_{1}, \ldots, \tau_{k}(\omega)=m_{k}\right\} \in \mathcal{F}_{m_{k}}$ for all $m_{k}>\cdots>m_{1} \geq 1$

2. $1 \leq \tau_{1}<\cdots<\tau_{k}<+\infty(\mathbf{P}-$ a.s. $)$.

The following condition is needed for the existence of all considered expectations (see $[3,4,29]$ ). There is a number of other sufficient conditions that ensure the existence of the expectations; for example, assumption Equation (13) in Theorem 2 in [30] and several different conditions discussed in [31].

$$
\mathbf{E}\left(\sup _{n} \mathbf{E}_{n}\left(\sup Z_{m_{1}, \ldots, m_{k}}^{+}\right)\right)<+\infty,
$$

where $x^{+}=\max \{x, 0\}, \mathbf{E}_{n}$ is the conditional expectations relative to the $\sigma$-algebras $\mathcal{F}_{n}$.

Now we can define optimal multiple stopping rules (see [3]).

Definition 2. Let $S_{m}$ be a class of multiple stopping rules $\tau=\left(\tau_{1}, \ldots \tau_{k}\right)$ such that $\tau_{1} \geq m$ (P-a.s.). The function

$$
v_{m}=\sup _{\tau \in S_{m}} \mathbf{E} Z_{\tau}
$$

is called the m-value. In particular, if $m=1$ then $v=v_{1}$ is the expected gain (or the value of the game).

In the other words, $S_{m}$ is the set of all multiple stopping times with respect to $\left\{\mathcal{F}_{n}\right\}$ such that $\left(\tau_{1}, \ldots, \tau_{k}\right) \in S_{m}$ if $\tau_{1} \geq m$.

Definition 3. A multiple stopping rule $\tau^{*} \in S_{m}$ is called an optimal multiple stopping rule in $S_{m}$ if $\mathbf{E} Z_{\tau^{*}}$ exists and $\mathrm{EZ}_{\tau^{*}}=v_{m}$.

The existence conditions and the structure of an optimal multiple stopping rule as well as further details of the theory of multiple optimal stopping rules can be found in [3-5,18].

\subsection{Finite Case}

Assume now that we observe a finite sequence of random variables $y_{1}, y_{2}, \ldots, y_{N}$. Let

$$
\begin{array}{r}
\left\{Z_{m_{1}, \ldots, m_{k}}, 1 \leq m_{1} \leq N_{1}, m_{1}<m_{2} \leq N_{2}\left(m_{1}\right), \ldots,\right. \\
\left.m_{k-1}<m_{k} \leq N_{k}\left(m_{1}, \ldots, m_{k-1}\right)\right\}
\end{array}
$$

be a family of random variables, where $N_{1}, N_{i}(\cdot)(i=2, \ldots, k)$ are natural numbers, which represent the latest possible times we are allowed to stop. For example, $N_{1}=N-k+1, N_{2}=N-k+2, \ldots, N_{k}=N$. The sequences $\left\{V_{m_{1}, \ldots, m_{i}}\right\}$ and $\left\{X_{m_{1}, \ldots, m_{i}}\right\}, i=1,2, \ldots, k$, are needed for constructing the multiple stopping rules. They can be defined by backward induction from the following recurrent equations; see formulas (7), (10) and (11) in [3], further details can be found in [2,29]:

$$
\begin{aligned}
& V_{m_{1}, \ldots, m_{i-1}, N_{i}\left(m_{1}, \ldots, m_{i-1}\right)}=X_{m_{1}, \ldots, m_{i-1}, N_{i}\left(m_{1}, \ldots, m_{i-1}\right)}, \\
& V_{m_{1}, \ldots, m_{i}}=\max \left\{X_{m_{1}, \ldots, m_{i}}, \mathbf{E}_{m_{i}} V_{m_{1}, \ldots, m_{i-1}, m_{i}+1}\right\}, \\
& X_{m_{1}, \ldots, m_{i-1}}=\mathbf{E}_{m_{i-1}} V_{m_{1}, \ldots, m_{i-1}, m_{i-1}+1}, \\
& X_{m_{1}, \ldots, m_{k}}=Z_{m_{1}, \ldots, m_{k}}
\end{aligned}
$$

for $1 \leq m_{1} \leq N_{1}, m_{i-1}<m_{i} \leq N_{i}\left(m_{1}, \ldots, m_{i-1}\right), i=2, \ldots, k$. After having observed the first $m_{i}$ observations, $X_{m_{1}, \ldots, m_{i}}$ can be interpreted as the conditional expected gain if we stop at time $m_{i}$ and use optimal stopping times for the later stages; $V_{m_{1}, \ldots, m_{i}}$ represents the maximum gain that is possible to receive at time $m_{i}$. If $m_{i}=N_{i}\left(m_{1}, \ldots, m_{i-1}\right)$, then the gain is $V_{m_{1}, \ldots, m_{i-1}, N_{i}\left(m_{1}, \ldots, m_{i-1}\right)}=$ 
$X_{m_{1}, \ldots, m_{i-1}, N_{i}\left(m_{1}, \ldots, m_{i-1}\right)}$. If $m_{i}<N_{i}\left(m_{1}, \ldots, m_{i-1}\right)$, we can either stop or continue. If we stop, the gain is $X_{m_{1}, \ldots, m_{i}}$, and if we continue, the gain is $\mathbf{E}_{m_{i}} V_{m_{1}, \ldots, m_{i-1}, m_{i}+1}$.

In [3], Theorem 1 and Theorem 4 give the existence conditions and the structure of an optimal multiple stopping rule $\tau^{*}$ in $S_{m}$ and the characterization of the $m$-value $v_{m}$ by means of the sequence $\left\{V_{m}\right\}$, respectively. Using these results, we obtain the optimal multiple stopping rule $\tau^{*}$ and the value $v_{m}$.

The following theorem gives a multiple optimal stopping rule and the expected gain for sums of independent random variables. It presents a solution of the problem formulated in Section 1 if all $C_{n}=1, n=1, \ldots, N$, which implies that $c_{m_{1}}=\cdots=c_{m_{k}}=1$ and $k=K$, that is, $c_{n}$ are known constants. The more complicated problem, when $c_{n}$ are not fixed, $0 \leq c_{n} \leq C_{n}$, is solved in Section 3 .

Theorem 1 ([18]). Let $y_{1}, y_{2}, \ldots, y_{N}$ be a sequence of independent random variables with known distribution functions $F_{1}, F_{2}, \ldots, F_{N}$,

$$
Z_{(m)_{k}}=y_{m_{1}}+y_{m_{2}}+\cdots+y_{m_{k}}
$$

Let $v^{L, l}$ be the expected gain with $l, l \leq k$, stops and $L, L \leq N$, steps. If there exist $\mathbf{E} y_{1}, \mathbf{E} y_{2}, \ldots, \mathbf{E} y_{N}$, then the value $v=v^{N, k}$, where

$$
\begin{aligned}
v^{n, 1}= & \mathbf{E}\left(\max \left\{y_{N-n+1}, v^{n-1,1}\right\}\right), 1 \leq n \leq N, v^{0,1}=-\infty \\
v^{n, k-i+1}= & \mathbf{E}\left(\max \left\{v^{n-1, k-i}+y_{N-n+1}, v^{n-1, k-i+1}\right\}\right), k-i+1 \leq n \leq N \\
& v^{k-i, k-i+1}=-\infty, i=k-1, \ldots, 1 .
\end{aligned}
$$

We put

$$
\begin{aligned}
\tau_{1}^{*}= & \min \left\{m_{1}: 1 \leq m_{1} \leq N-k+1, y_{m_{1}} \geq v^{N-m_{1}, k}-v^{N-m_{1}, k-1}\right\} \\
\tau_{i}^{*}=\min \left\{m_{i}: \tau_{i-1}^{*}<m_{i} \leq N-k+i, y_{m_{i}} \geq v^{N-m_{i}, k-i+1}-v^{N-m_{i}, k-i}\right\}, & i=2, \ldots, k-1, \\
\tau_{k}^{*}=\min \left\{m_{k}: \tau_{k-1}^{*}<m_{k} \leq N, y_{m_{k}} \geq v^{N-m_{k}, 1}\right\}, &
\end{aligned}
$$

then $\tau^{*}=\left(\tau_{1}^{*}, \ldots, \tau_{k}^{*}\right)$ is the optimal multiple stopping rule.

\section{Main Result}

Theorem 1 gives a multiple optimal stopping rule and the expected gain for a problem with a fixed number of stops $k$. However, in our initial problem, the $k$ is not fixed since at time $n$ we can sell $0,1 \ldots$ or $C_{n}$ objects, $C_{n}$ are known positive constants, $n=1,2, \ldots, N$. Obviously, our decision may affect the remaining number of stops required to sell all of the $K$ objects. In this case, $c_{n}$ are $\mathcal{F}_{n}$-measurable random variables taking values from $\left\{0,1, \ldots, C_{n}\right\}$, where $\mathcal{F}_{n}=\sigma\left(y_{1}, \ldots, y_{n}\right)$.

Let us consider an extended version of the problem. We observe a sequence of random variables $\tilde{y}_{1}, \tilde{y}_{2}, \ldots, \tilde{y}_{S_{N}}, S_{N}=C_{1}+\cdots+C_{N}$, where

$$
\tilde{y}_{j}= \begin{cases}y_{1}, & 1 \leq j \leq S_{1} \\ y_{2}, & S_{1}+1 \leq j \leq S_{2} \\ \vdots & \\ y_{N}, & S_{N-1}+1 \leq j \leq S_{N}\end{cases}
$$

$\left\{S_{n}\right\}_{n=1}^{N}$ is a cumulative sum, $S_{n}=C_{1}+\cdots+C_{n}$. In the other words, we observe the following sequence

$$
\underbrace{y_{1}, \ldots, y_{1}}_{C_{1}}, \underbrace{y_{2}, \ldots, y_{2}}_{C_{2}}, \ldots, \underbrace{y_{N}, \ldots, y_{N}}_{C_{N}} .
$$


The following theorem gives a multiple optimal stopping rule and the expected gain for the stopping problem of the extended sequence when we are allowed to stop $K$ times and we receive the gain

$$
Z_{m_{1}, m_{2}, \ldots, m_{K}}=\tilde{y}_{m_{1}}+\tilde{y}_{m_{2}}+\cdots+\tilde{y}_{m_{K}} .
$$

Theorem 2. Let $\tilde{y}_{1}, \tilde{y}_{2}, \ldots, \tilde{y}_{S_{N}}$ be a sequence of random variables as in Equation (8) with the gain function Equation (9). Let $v^{L, l}$ be the expected gain with $l, l \leq K$, stops and $L, L \leq S_{N}$, steps. If there exist $\mathbf{E} \tilde{y}_{1}, \mathbf{E} \tilde{y}_{2}, \ldots, \mathbf{E} \tilde{y}_{S_{N}}$, then the value $v=v^{S_{N}, K}$, where

$$
\begin{aligned}
& v^{S_{N}-n+1,1}= \begin{cases}\mathbf{E}\left(\max \left\{\tilde{y}_{n}, v^{S_{N}-n, 1}\right\}\right), & n-1 \in\left\{S_{1}, \ldots, S_{N-1}\right\}, \\
\max \left\{\tilde{y}_{n}, v^{S_{N}-n, 1}\right\}, & \text { otherwise. }\end{cases} \\
& 1 \leq n \leq S_{N}, v^{0,1}=-\infty,
\end{aligned}
$$

We put

$$
\begin{aligned}
& \tau_{1}^{*}=\min \left\{m_{1}: 1 \leq m_{1} \leq S_{N}-K+1, \tilde{y}_{m_{1}} \geq v^{S_{N}-m_{1}, K}-v^{S_{N}-m_{1}, K-1}\right\}, \\
& \tau_{i}^{*}=\min \left\{m_{i}: \tau_{i-1}^{*}<m_{i} \leq S_{N}-K+i, \tilde{y}_{m_{i}} \geq v^{S_{N}-m_{i}, K-i+1}-v^{S_{N}-m_{i}, K-i}\right\}, \\
& \tau_{K}^{*}=\min \left\{m_{K}: \tau_{K-1}^{*}<m_{K} \leq S_{N}, \tilde{y}_{m_{K}} \geq v^{S_{N}-m_{K}, 1}\right\} . i=2, \ldots, K-1,
\end{aligned}
$$

then $\tau^{*}=\left(\tau_{1}^{*}, \ldots, \tau_{K}^{*}\right)$ is the optimal multiple stopping rule.

Proof. From Equation (5) we obtain

$$
\begin{aligned}
V_{m_{1}, \ldots, m_{K}} & =\max \left\{X_{m_{1}, \ldots, m_{K}}, \mathbf{E}_{m_{K}} V_{m_{1}, \ldots, m_{K-1}, m_{K}+1}\right\} \\
& =\max \left\{\tilde{y}_{m_{1}}+\cdots+\tilde{y}_{m_{K}}, \mathbf{E}_{m_{K}} V_{m_{1}, \ldots, m_{K-1}, m_{K}+1}\right\} \\
& =\max \left\{\tilde{y}_{m_{K}}, v^{S_{N}-m_{K}, 1}\right\}+\tilde{y}_{m_{1}}+\cdots+\tilde{y}_{m_{K-1}} .
\end{aligned}
$$

For $m_{K-1}<m_{K}<S_{N}$,

$$
\begin{aligned}
v^{S_{N}-m_{K}+1,1} & =\mathbf{E}_{m_{K}-1}\left(\max \left\{\tilde{y}_{m_{K}}, v^{S_{N}-m_{K}, 1}\right\}\right) \\
& = \begin{cases}\mathbf{E}\left(\max \left\{\tilde{y}_{m_{K}}, v^{S_{N}-m_{K}, 1}\right\}\right), & m_{K}-1 \in\left\{S_{1}, \ldots, S_{N-1}\right\}, \\
\max \left\{\tilde{y}_{m_{K}}, v^{S_{N}-m_{K}, 1}\right\}, & \text { otherwise. }\end{cases}
\end{aligned}
$$

For $m_{K}=S_{N}$,

$$
v^{S_{N}-m_{K}+1,1}=v^{1,1}= \begin{cases}\mathbf{E} \tilde{y}_{S_{N}}, & \text { if } m_{K}=S_{N-1}+1 \\ \tilde{y}_{S_{N}} & \text { otherwise }\end{cases}
$$

since $v^{0,1}=-\infty$.

In the other words, if $C_{N}=S_{N}-S_{N-1}=1$,

$$
v^{C_{N}, 1}=v^{1,1}=\mathbf{E} \tilde{y}_{S_{N}}=\mathbf{E} y_{N} .
$$


If $C_{N}=S_{N}-S_{N-1}>1$,

$$
v^{1,1}=v^{2,1}=\cdots=v^{C_{N}-1,1}=y_{N},
$$

whereas

$$
v^{C_{N}, 1}=\mathbf{E} \tilde{y}_{S_{N-1}+1}=\mathbf{E} y_{N} .
$$

According to Theorem 1 [3], if $\tilde{y}_{m_{K}} \geq v^{S_{N}-m_{K}, 1}$, then we need to stop at time $m_{K}$. Therefore, should we decide to stop at time $S_{n-1}+1$, that is, in the beginning of the series

$$
\underbrace{y_{n}, \ldots, y_{n}}_{C_{n}}
$$

we will also stop at times $S_{n-1}+2, \ldots, S_{n}$. This implies that we will be able to sell $C_{n}$ objects.

From Equations (6) and (10) we have

$$
\begin{aligned}
X_{m_{1}, \ldots, m_{K-1}} & =\mathbf{E}_{m_{K-1}} V_{m_{1}, \ldots, m_{K-1}, m_{K-1}+1} \\
& =\mathbf{E}_{m_{K-1}}\left(\max \left\{\tilde{y}_{m_{K-1}+1}, v^{S_{N}-m_{K-1}-1,1}\right\}+\tilde{y}_{m_{1}}+\cdots+\tilde{y}_{m_{K-1}}\right) \\
& =v^{S_{N}-m_{K-1}, 1}+\tilde{y}_{m_{1}}+\cdots+\tilde{y}_{m_{K-1}}
\end{aligned}
$$

since

$$
\begin{aligned}
& \mathbf{E}_{m_{K-1}}\left(\max \left\{\tilde{y}_{m_{K-1}+1}, v^{S_{N}-m_{K-1}-1,1}\right\}\right. \\
& = \begin{cases}\mathbf{E}\left(\max \left\{\tilde{y}_{m_{K-1}+1}, v^{S_{N}-m_{K-1}-1,1}\right\}\right), & m_{K-1} \in\left\{S_{1}, \ldots, S_{N-1}\right\}, \\
\max \left\{\tilde{y}_{m_{K-1}+1}, v^{S_{N}-m_{K-1}-1,1}\right\}, & \text { otherwise. }\end{cases}
\end{aligned}
$$

In the same way,

$$
\begin{aligned}
V_{m_{1}, \ldots, m_{i}} & =\max \left\{X_{m_{1}, \ldots, m_{i}}, \mathbf{E}_{m_{i}} V_{m_{1}, \ldots, m_{i-1}, m_{i}+1}\right\} \\
& =\max \left\{v^{S_{N}-m_{i}, K-i}+\tilde{y}_{m_{1}}+\cdots+\tilde{y}_{m_{i}}, \mathbf{E}_{m_{i}} V_{m_{1}, \ldots, m_{i-1}, m_{i}+1}\right\} \\
& =\max \left\{v^{S_{N}-m_{i}, K-i}+\tilde{y}_{m_{i}}, v^{S_{N}-m_{i}, K-i+1}\right\} \\
& +\tilde{y}_{m_{1}}+\cdots+\tilde{y}_{m_{i-1}}
\end{aligned}
$$

where, for $m_{i-1}<m_{i}<S_{N}-K+i$,

$$
\begin{aligned}
& v^{S_{N}-m_{i}+1, K-i+1}=\mathbf{E}_{m_{i}-1}\left(\max \left\{v^{S_{N}-m_{i}, K-i}+\tilde{y}_{m_{i}}, v^{S_{N}-m_{i}, K-i+1}\right\}\right) \\
& = \begin{cases}\mathbf{E}\left(\operatorname { m a x } \left\{v^{S_{N}-m_{i}, K-i}+\tilde{y}_{m_{i}},\right.\right. & m_{i}-1 \in\left\{S_{1}, \ldots, S_{N-1}\right\}, \\
\left.\left.v^{S_{N}-m_{i}, K-i+1}\right\}\right), & \text { otherwise } \\
\max \left\{v^{S_{N}-m_{i}, K-i}+\tilde{y}_{m_{i}},\right. & \\
\left.v^{S_{N}-m_{i}, K-i+1}\right\}, & \end{cases}
\end{aligned}
$$

with $v^{K-i, K-i+1}=-\infty, i=K-1, K-2, \ldots, 2$.

Similarly, for $i=K-1, K-2, \ldots, 2$,

$$
\begin{aligned}
X_{m_{1}, \ldots, m_{i-1}}= & \mathbf{E}_{m_{i-1}} V_{m_{1}, \ldots, m_{i-1}, m_{i-1}+1} \\
= & \mathbf{E}_{m_{i-1}}\left(\max \left\{v^{S_{N}-m_{i-1}-1, K-i}+\tilde{y}_{m_{i-1}+1}, v^{S_{N}-m_{i-1}-1, K-i+1}\right\}\right. \\
& \left.+\tilde{y}_{m_{1}}+\cdots+\tilde{y}_{m_{i-1}}\right) \\
= & v^{S_{N}-m_{i-1}, K-i+1}+\tilde{y}_{m_{1}}+\cdots+\tilde{y}_{m_{i-1}}
\end{aligned}
$$


since

$$
\begin{aligned}
& \mathbf{E}_{m_{i-1}}\left(\max \left\{v^{S_{N}-m_{i-1}-1, K-i}+\tilde{y}_{m_{i-1}+1}, v^{S_{N}-m_{i-1}-1, K-i+1}\right\}\right) \\
& =\left\{\begin{array}{l}
\mathbf{E}\left(\operatorname { m a x } \left\{v^{S_{N}-m_{i-1}-1, K-i}+\tilde{y}_{m_{i-1}+1},\right.\right. \\
\left.\left.v^{S_{N}-m_{i-1}-1, K-i+1}\right\}\right), \\
\max \left\{v^{S_{N}-m_{i-1}-1, K-i}+\tilde{y}_{m_{i-1}+1},\right. \\
\left.v^{S_{N}-m_{i-1}-1, K-i+1}\right\},
\end{array} \quad\right. \text { otherwise. }
\end{aligned}
$$

Finally,

$$
\begin{aligned}
V_{m_{1}} & =\max \left\{X_{m_{1}}, \mathbf{E}_{m_{1}} V_{m_{1}+1}\right\} \\
& =\max \left\{v^{S_{N}-m_{1}, K-1}+\tilde{y}_{m_{1}}, \mathbf{E}_{m_{1}} V_{m_{1}+1}\right\} \\
& =\max \left\{v^{S_{N}-m_{1}, K-1}+\tilde{y}_{m_{1}}, v^{S_{N}-m_{1}, K}\right\}
\end{aligned}
$$

where, for $1 \leq m_{1}<S_{N}-K+1$,

$$
\begin{aligned}
& v^{S_{N}-m_{1}+1, K}=\mathbf{E}_{m_{1}-1}\left(\max \left\{v^{S_{N}-m_{1}, K-1}+\tilde{y}_{m_{1}}, v^{S_{N}-m_{1}, K}\right\}\right) \\
& = \begin{cases}\mathbf{E}\left(\max \left\{v^{S_{N}-m_{1}, K-1}+\tilde{y}_{m_{1}}, v^{S_{N}-m_{1}, K}\right\}\right), & m_{1}-1 \in\left\{S_{1}, \ldots, S_{N-1}\right\}, \\
\max \left\{v^{S_{N}-m_{1}, K-1}+\tilde{y}_{m_{1}}, v^{S_{N}-m_{1}, K}\right\}, & \text { otherwise }\end{cases}
\end{aligned}
$$

with $v^{K-1, K}=-\infty$.

Taking into account Theorem 4 [3], we obtain $v=v^{S_{N}, K}$.

Now, using Theorem 1 [3], Equations (10)-(13) we obtain the optimal multiple stopping rule $\tau^{*}=\left(\tau_{1}^{*}, \ldots, \tau_{K}^{*}\right)$

$$
\begin{aligned}
\tau_{1}^{*}= & \min \left\{m_{1}: 1 \leq m_{1} \leq S_{N}-K+1, \tilde{y}_{m_{1}} \geq v^{S_{N}-m_{1}, K}-v^{S_{N}-m_{1}, K-1}\right\}, \\
\tau_{i}^{*}= & \min \left\{m_{i}: \tau_{i-1}^{*}<m_{i} \leq S_{N}-K+i, \tilde{y}_{m_{i}} \geq v^{S_{N}-m_{i}, K-i+1}-v^{S_{N}-m_{i}, K-i}\right\}, \\
\tau_{K}^{*}= & \min \left\{m_{K}: \tau_{K-1}^{*}<m_{K} \leq S_{N}, \tilde{y}_{m_{K}} \geq v^{S_{N}-m_{K}, 1}\right\} . K-1,
\end{aligned}
$$

This completes the proof.

Theorem 2 allows us to write down $c_{n}$ in an explicit form, For example,

$$
\begin{aligned}
& c_{1}= \begin{cases}\min \left\{C_{1}, K\right\}, & y_{1} \geq v^{S_{N}-1, K}-v^{S_{N}-1, K-1}, \\
0, & y_{1}<v^{S_{N}-1, K}-v^{S_{N}-1, K-1}\end{cases} \\
& c_{2}= \begin{cases}\min \left\{C_{2}, K\right\}, & y_{1}<v^{S_{N}-1, K}-v^{S_{N}-1, K-1} \\
\min \left\{C_{2}, K-c_{1}\right\}, & y_{2} \geq v^{S_{N}-C_{1}-1, K}-v^{S_{N}-C_{1}-1, K-1} \\
& y_{2} \geq v^{S_{N}-1, K}-v^{S_{N}-1, K-1} \\
0, & \text { otherwise. }\end{cases}
\end{aligned}
$$

We can see that $c_{1}$ depends on $y_{1} ; c_{2}$ depends on $y_{1}, y_{2}$ and $c_{1}$. Similarly, we can obtain expressions for $c_{3}, c_{4}, \ldots, c_{N}$ with $c_{n}=\min \left\{C_{n}, K-s\right\}$ on $\left\{c_{1}+\cdots+c_{n-1}=s\right\}, s \in\{0,1, \ldots, K\}$. Note that the actual number of stops $k=\sum_{n=1}^{N} I\left(c_{n}>0\right)$.

Corollary 1. If $C_{1}=\cdots=C_{N}=1$, the extended sequence $\tilde{y}_{1}, \tilde{y}_{2}, \ldots, \tilde{y}_{S_{N}}$ coincides with the original sequence $y_{1}, y_{2}, \ldots, y_{N}$. So we consider the stopping problem of the original sequence with $K$ stops allowed. Since $m_{i} \in\left\{S_{1}, \ldots, S_{N}\right\}=\{1,2, \ldots, N\}, i=1,2, \ldots, K$, the values $v^{L, l}, l \leq K, L \leq N$, are calculated in the 
same way as in Theorem 1, which gives the multiple optimal stopping rule $\tau^{*}=\left(\tau_{1}^{*}, \ldots, \tau_{K}^{*}\right)$. If we stop at time $n$, then $c_{n}=1$.

Corollary 2. If $C_{1}=\cdots=C_{N}=C \geq 2, K=k C$, where $C, K, k$ are natural numbers, then, according to the optimal rule, if we decide to stop at some time, we should sell all of the C objects (see [1]). In the other words, we can consider a reduced problem with a gain

$$
Z_{m_{1}, m_{2}, \ldots, m_{k}}^{r}=y_{m_{1}}+y_{m_{2}}+\cdots+y_{m_{k}}
$$

where $Z_{m_{1}, m_{2}, \ldots, m_{k}}=C Z_{m_{1}, m_{2}, \ldots, m_{k}}^{r}$ is the initial gain Equation (1). If we stop at time $n$, then $c_{n}=C$.

Corollary 3. If $\min \left\{C_{1}, \ldots, C_{N}\right\} \geq K$, the problem Equation (1) is reduced to a problem with 1 stop and a gain $Z_{m_{1}}^{r}=y_{m_{1}}, Z_{m_{1}}=K Z_{m_{1}}^{r}$. This means that the optimal rule will require us to stop once and sell all of the $K$ objects (see [1]), that is, $c_{n}=K$ if we decide to stop at time $n$.

\section{Examples}

\subsection{Uniform Distribution}

Let $y_{1}, y_{2}, \ldots, y_{N}$ be a sequence of independent random variable having uniform distribution $\mathrm{U}(a, b), a, b$ are fixed numbers. From Example 1 [1] we have

$$
\begin{aligned}
& v^{n, 1}=\left(v^{n-1,1}-a\right)^{2} /(2(b-a))+(a+b) / 2, \\
& v^{n, k}=\left(v^{n-1, k}-v^{n-1, k-1}-a\right)^{2} /(2(b-a))+(a+b) / 2+v^{n-1, k-1},
\end{aligned}
$$

where $v^{0,1}=a, v^{k, k+1}=a+k(a+b) / 2,1 \leq n \leq N$.

In Table 1 (see [1]), we present the values $v^{L, l}$ with $l(l \leq 7)$ stoppings and $L(L \leq 10)$ steps for the choice $a=0, b=1$.

Table 1. The values for the uniform distribution $\mathrm{U}(0,1)$.

\begin{tabular}{cccccccc}
\hline$L$ & $v^{L, 1}$ & $v^{L, 2}$ & $v^{L, 3}$ & $v^{L, 4}$ & $v^{L, 5}$ & $v^{L, 6}$ & $v^{L, 7}$ \\
\hline 1 & 0.5000 & & & & & & \\
2 & 0.6250 & 1.0000 & & & & & \\
3 & 0.6953 & 1.1953 & 1.5000 & & & & \\
4 & 0.7417 & 1.3203 & 1.7417 & 2.0000 & & & \\
5 & 0.7751 & 1.4091 & 1.9091 & 2.2751 & 2.5000 & & \\
6 & 0.8004 & 1.4761 & 2.0341 & 2.4761 & 2.8004 & 3.0000 & \\
7 & 0.8203 & 1.5287 & 2.1318 & 2.6318 & 3.0287 & 3.3203 & 3.5000 \\
8 & 0.8364 & 1.5712 & 2.2105 & 2.7568 & 3.2105 & 3.5712 & 3.8364 \\
9 & 0.8498 & 1.6064 & 2.2756 & 2.8597 & 3.3597 & 3.7756 & 4.1064 \\
10 & 0.8611 & 1.6360 & 2.3303 & 2.9462 & 3.4847 & 3.9462 & 4.3303 \\
\hline
\end{tabular}

Suppose that $N=5, K=2$, and $\left(C_{1}, C_{2}, C_{3}, C_{4}, C_{5}\right)=(2,3,4,3,2)$. Then, according to Corollary 3 , we should stop one time and sell both objects. In this case the expected gain is $2 v^{5,1}=2 \cdot 0.7751=1.5502$.

We generated 10,000 sequences of length $N=5$ from $\mathrm{U}(0,1)$ and applied the rules defined as follows:

Rule 1 (optimal): the optimal stopping rule (with 1 stop);

Rule 2 (sub-optimal): the optimal double stopping rule (with $K=2$ stops);

Rule 3 (deterministic): always sell at the beginning $\left(m_{1}=1\right)$ of the time interval;

Rule 4 (random): stop randomly at $m_{1}$ and $m_{2}$, subject to $1 \leq m_{1}<m_{2} \leq 5$.

Table 2 displays the average gains of the rules indicating that Rule 1 (the optimal rule) outperforms the other three rules. 
Table 2. The average gains for different rules: optimal (Rule 1), sub-optimal (Rule 2), deterministic (Rule 3) and random (Rule 4).

\begin{tabular}{lcccc}
\hline & Rule 1 & Rule 2 & Rule 3 & Rule 4 \\
\hline Average gain & 1.5460 & 1.4109 & 1.0073 & 1.0051 \\
\hline
\end{tabular}

\subsection{Exponential Distribution}

Suppose independent random variables $y_{1}, y_{2}, \ldots, y_{N}$ are identically distributed with an exponential distribution $\operatorname{Exp}(\beta)$. Its probability density function is

$$
f(x)=\frac{1}{\beta} \exp \left(-\frac{x}{\beta}\right), x>0, \beta>0 .
$$

Suppose that $C_{1}=C_{2}=\cdots=C_{N}=1$, then, according to Corollary 1 , the values $v^{L, l}, l \leq K$, $L \leq N$ and the multiple optimal stopping rule $\tau^{*}=\left(\tau_{1}^{*}, \ldots, \tau_{K}^{*}\right)$ can be found from Theorem 1 .

We have

$$
\begin{aligned}
v^{n, 1} & =\mathbf{E}\left(\max \left\{y_{N-n+1}, v^{n-1,1}\right\}\right) \\
& =\int_{0}^{\infty} \max \left\{x, v^{n-1,1}\right\} f(x) d x \\
& =\int_{0}^{v^{n-1,1}} v^{n-1,1} f(x) d x+\int_{v^{n-1,1}}^{\infty} x f(x) d x \\
& =v^{n-1,1}-v^{n-1,1} \exp \left(-\frac{v^{n-1,1}}{\beta}\right)+\left(\beta+v^{n-1,1}\right) \exp \left(-\frac{v^{n-1,1}}{\beta}\right) \\
& =v^{n-1,1}+\beta \exp \left(-\frac{v^{n-1,1}}{\beta}\right), \quad 1 \leq n \leq N, v^{0,1}=0
\end{aligned}
$$

Likewise,

$$
\begin{aligned}
v^{n, k-i+1} & =\mathbf{E}\left(\max \left\{v^{n-1, k-i}+y_{N-n+1}, v^{n-1, k-i+1}\right\}\right) \\
& =\mathbf{E}\left(\max \left\{y_{N-n+1}, v^{n-1, k-i+1}-v^{n-1, k-i}\right\}\right)+v^{n-1, k-i} \\
& =\int_{0}^{\infty} \max \{x, c\} f(x) d x+v^{n-1, k-i} \\
& =\int_{0}^{c} c f(x) d x+\int_{c}^{\infty} x f(x) d x+v^{n-1, k-i} \\
& =v^{n-1, k-i+1}+\beta \exp \left(-\frac{v^{n-1, k-i+1}-v^{n-1, k-i}}{\beta}\right)
\end{aligned}
$$

where $c=v^{n-1, k-i+1}-v^{n-1, k-i}, k-i+1 \leq n \leq N, v^{k-i, k-i+1}=0, i=k-1, \ldots, 1$.

Table 3 presents the values $v^{L, l}$ with $l(l \leq 8)$ stoppings and $L(L \leq 8)$ steps for the choice $\beta=1$.

Table 3. The values for the exponential distribution $\operatorname{Exp}(1)$.

\begin{tabular}{ccccccccc}
\hline$L$ & $v^{L, 1}$ & $v^{L, 2}$ & $v^{L, 3}$ & $v^{L, 4}$ & $v^{L, 5}$ & $v^{L, 6}$ & $v^{L, 7}$ & $v^{L, 8}$ \\
\hline 1 & 1.0000 & & & & & & & \\
2 & 1.3679 & 2.0000 & & & & & & \\
3 & 1.6225 & 2.5315 & 3.0000 & & & & & \\
4 & 1.8199 & 2.9344 & 3.6259 & 4.0000 & & & & \\
5 & 1.9820 & 3.2625 & 4.1267 & 4.6879 & 5.0000 & & & \\
6 & 2.1198 & 3.5404 & 4.5481 & 5.2585 & 5.7319 & 6.0000 & & \\
7 & 2.2398 & 3.7819 & 4.9132 & 5.7499 & 6.3548 & 6.7648 & 7.0000 & \\
8 & 2.3463 & 3.9959 & 5.2358 & 6.1830 & 6.9009 & 7.4284 & 7.7905 & 8.0000 \\
\hline
\end{tabular}


For example, if $N=8$ and $K=2$, then the expected gain $v=v^{8,2}=3.9959$ and the optimal multiple stopping rule $\tau^{*}=\left(\tau_{1}^{*}, \tau_{2}^{*}\right)$ :

$$
\begin{aligned}
& \tau_{1}^{*}=\min \left\{m_{1}: 1 \leq m_{1} \leq 7, y_{m_{1}} \leq v^{8-m_{1}, 2}-v^{8-m_{1}, 1}\right\}, \\
& \tau_{2}^{*}=\min \left\{m_{2}: \tau_{1}^{*}<m_{2} \leq 8, y_{m_{2}} \geq v^{8-m_{2}, 1}\right\} .
\end{aligned}
$$

\section{Discussion}

In this paper, we have considered one of well-known problems in the decision making literature, an asset selling problem. While most of the literature on the subject has a focus on selling a single object, there are applications, which can be better modelled under the assumption of selling mutiple objects. For example, a seller wants to sell more than one object over a fixed period of time (say, a month). Many existing asset selling models allow either single offer or a fixed number of offers per time period (for example, per day). In contrast, the understanding of multiple selling problems when a number of offers per time period is not fixed has been limited so far. In this work, we have presented a novel sequential problem of selling several identical assets with a variable number of offers per time period. The model considered here assumes independence of sequentially observed random variables, generalisation to a case of dependent random variables is a matter for our further research. An important feature of the mutiple selling problem considered in the paper is that, since the number of the objects that can be sold at any time is a random variable, the proposed model is much more complicated compare to models with a fixed rate of offers. We have derived an optimal sequential rule and the expected gain for the multi-asset selling problem with a finite horizon. We have provided examples illustrating how the optimal procedure work.

Funding: This research received no external funding.

Acknowledgments: The author is grateful to Anna Krasnosielska-Kobos for her comments on the manuscript, and to the referees for helpful remarks.

Conflicts of Interest: The author declares no conflict of interest.

\section{References}

1. Sofronov, G. An optimal sequential procedure for a multiple selling problem with independent observations. Eur. J. Oper. Res. 2013, 225, 332-336. [CrossRef]

2. Chow, Y.S.; Robbins, H.; Siegmund, D. Great Expectations: The Theory of Optimal Stopping; Houghton Mifflin: Boston, MA, USA, 1971.

3. Nikolaev, M.L. Generalized sequential procedures. Lith. Math. J. 1979, 19, 318-325. [CrossRef]

4. Nikolaev, M.L. Test for optimality of a generalized sequence of procedures. Lith. Math. J. 1981, 21, $253-258$. [CrossRef]

5. Nikolaev, M.L. On optimal multiple stopping of Markov sequences. Theory Probab. Its Appl. 1999, 43, $298-306$. [CrossRef]

6. Lippman, S.A.; McCall, J.J. The economics of job search: A survey. Econ. Inq. 1976, 14, 155-189. [CrossRef]

7. Stein, W.E.; Seale, D.A.; Rapoport, A. Analysis of heuristic solutions to the best choice problem. Eur. J. Oper. Res. 2003, 151, 140-152. [CrossRef]

8. David, I. Explicit results for a class of asset-selling problems. Eur. J. Oper. Res. 1998, 110, 576-584. [CrossRef]

9. Righter, R. The stochastic sequential assignment problem with random deadlines. Probab. Eng. Inf. Sci. 1987, 1, 189-202. [CrossRef]

10. Seregina, T.V.; Ivashko, A.A.; Mazalov, V.V. Optimal stopping strategies in the game "the price is right". Proc. Steklov Inst. Math. 2019, 307, 127-141. [CrossRef]

11. Papastavrou, J.D.; Rajagopalan, S.; Kleywegt, A.J. The Dynamic and Stochastic Knapsack Problem with Deadlines. Manag. Sci. 1996, 42, 1706-1718. [CrossRef]

12. Karpowicz, A.; Szajowski, K. Double optimal stopping of a risk process. Stochastics 2007, 79, $155-167$. [CrossRef] 
13. Targino, R.S.; Peters, G.W.; Sofronov, G.; Shevchenko, P.V. Optimal exercise strategies for operational risk insurance via multiple stopping times. Methodol. Comput. Appl. Probab. 2017, 19, 487-518. [CrossRef]

14. Krasnosielska-Kobos, A. Multiple-stopping problems with random horizon. Optimization 2015, 64, $1625-1645$. [CrossRef]

15. Krasnosielska-Kobos, A. Construction of Nash equilibrium based on multiple stopping problem in multi-person game. Math. Methods Oper. Res. 2016, 83, 53-70. [CrossRef]

16. Stadje, W. A full information pricing problem for the sale of several identical commodities. Z. Oper. Res. 1990, 34, 161-181. [CrossRef]

17. Tanaka, T. Discrete Time Optimal Stopping Problems with Fractional Rewards. J. Inf. Optim. Sci. 2014, 35, 291-306. [CrossRef]

18. Nikolaev, M.L.; Sofronov, G.Y. A multiple optimal stopping rule for sums of independent random variables. Discret. Math. Appl. 2007, 17, 463-473. [CrossRef]

19. Sofronov, G.; Keith, J.M.; Kroese, D.P. An optimal sequential procedure for a buying-selling problem with independent observations. J. Appl. Probab. 2006, 43, 454-462. [CrossRef]

20. Sofronov, G.Y. A multiple optimal stopping rule for a buying-selling problem with a deterministic trend. Stat. Pap. 2016, 57, 1107-1119. [CrossRef]

21. Sofronov, G.Y. An optimal double stopping rule for a buying-selling problem. Methodol. Comput. Appl. Probab. 2020, 22, 1-12. [CrossRef]

22. Albright, S.C. A Bayesian approach to a generalized housing selling problem. Manag. Sci. 1977, 24, 432-440. [CrossRef]

23. MacQueen, J.; Miller, R.G. Optimal Persistence Policies. Oper. Res. 1960, 8, 362-380. [CrossRef]

24. Albright, S.C. Optimal Sequential Assignments with Random Arrival Times. Manag. Sci. 1974, $21,60-67$. [CrossRef]

25. Bruss, F.T.; Ferguson, T.S. Multiple buying or selling with vector offers. J. Appl. Probab. 1997, 34, 959-973. [CrossRef]

26. Rosenfield, D.B.; Shapiro, R.D.; Butler, D.A. Optimal strategies for selling an asset. Manag. Sci. 1983, 29, 1051-1061. [CrossRef]

27. David, I.; Levi, O. Asset-selling problems with holding costs. Int. J. Prod. Econ. 2001, 71, 317-321. [CrossRef]

28. Chun, Y.H.; Plante, R.D.; Schneider, H. Buying and selling an asset over the finite time horizon: A non-parametric approach. Eur. J. Oper. Res. 2002, 136, 106-120. [CrossRef]

29. Haggstrom, G.W. Optimal Sequential Procedures when More Than one Stop is Required. Ann. Math. Stat. 1967, 38, 1618-1626. [CrossRef]

30. Stadje, W. On multiple stopping rules. Optimization 1985, 16, 401-418. [CrossRef]

31. Kösters, H. A note on multiple stopping rules. Optimization 2004, 53, 69-75. [CrossRef] 